\title{
HISTORIA, IMPORTANCIA Y APLICACIONES DEL MÉTODO DE LECTURA DE PARAMENTOS
}

\author{
(HISTORY, IMPORTANCE AND APPLICATIONS OF THE STRATIGRAPHIC METHOD)
}

Roberto Parenti, Arqueólogo y Arquitecto

Fecha de recepción: 13-II-95

Dpto. de Arqueología e Historia del Arte

Universidad de Siena

ITALIA

\section{RESUMEN}

Después de más de diez años de experimentación sobre el análisis arquitectónico con un instrumento creado para la investigación arqueológica, en Italia se ha conseguido un balance y se han definido nuevas líneas de investigación que se refieren a campos especializados muy diferentes. No obstante se debe insistir en que, en los casos más atentos al avance de la investigación cientifica, la relación entre arqueólogos y arquitectos ocupados de la restauración se orienta hacia una colaboración cada vez más integrada. En las actuaciones ejemplares, la investigación continúa; pero, mientras, es preciso hacer didácticamente comprensibles los criterios y capacidades de este instrumento, con el fin de que puedan valerse de él muchos más investigadores, ya que el patrimonio arquitectónico, ya sea un monumento o la vivienda más modesta, es como un enorme registro que recoge en su estructura una gran cantidad de datos. Datos que la mayor parte de los investigadores deben poder descodificar con criterios uniformes y transmisibles, si queremos que no se pierda la gran masa de observaciones y que se conviertan en las informaciones necesarias para que avancen nuestros conocimientos

\section{SUMMARY}

After for over ten years of experimentations into architecture studies carried out by a tool settled in archaeological research, in Italy we draw up a balance dual we determine new utlines of research that apply at differented disciplinary limits. Neverthless we have to insist because the connection between archaeologist and architect engaged in the restoration field, tend towards a more strict collaboration. In the best situations the research is getting on, but we should make didactly comprehensible the principles and the capacity of the tools, so that it might be used by many operators, because the architectural heritage, either monumental one or a simple dwelling, it is like an enormous recorder that records a large amount of data. Those data must be understood by the operators, with an easy and useful tool, if we don't want lose the amount of remark and will change them into progress of knowledge.

\section{Las premisas teóricas}

Han pasado casi dos decenios desde que se desarrollaran en Italia las primeras experiencias pioneras sobre el método de análisis denominado estratigráfico y ya estamos en el punto en que podemos hacer una pausa y reflexionar; pausa necesaria para verificar los resultados obtenidos, extraer principios y renovar las energías que permitan mirar más allá, ampliar un panorama no comprometido con el inevitable corporativismo disciplinar y con el espíritu analítico necesario para afrontar los muy particulares aspectos que se encuentran en este recorrido ${ }^{1}$. Cada vez se distinguen con más claridad las líneas que indican el camino por el que deben proseguir nuestros trabajos, al mismo tiempo que aumenta la tentación de sondear nuevas posibilidades, de verificar ramales y conexiones que pueden parecer a primera vista carentes de efectos inmediatos, pero que, en cualquier caso, siempre merecen atención. Cuando en 1974 Tiziano Mannoni propuso registrar las características de la obra de fábrica con criterios lo más 


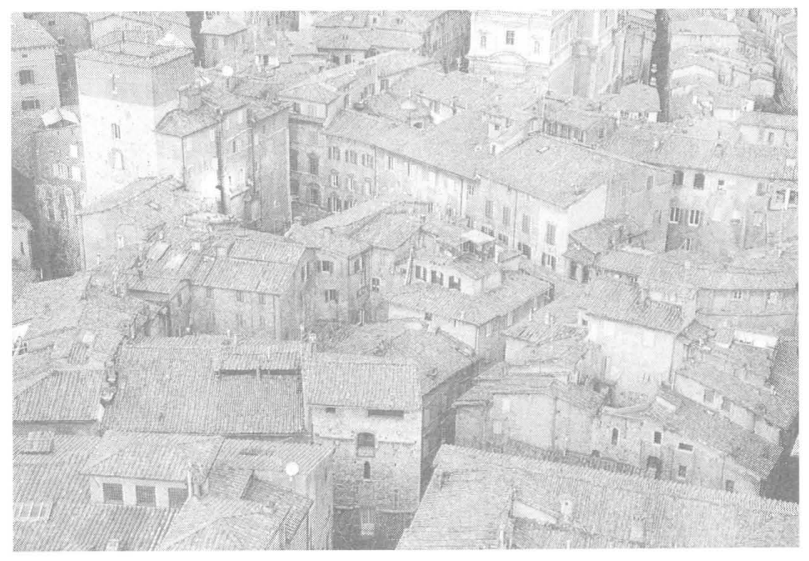

Fig.1.- Una vista del centro histórico de Siena que muestra la contimua intervención constructiva que se añade a las viviendas más antiguas.

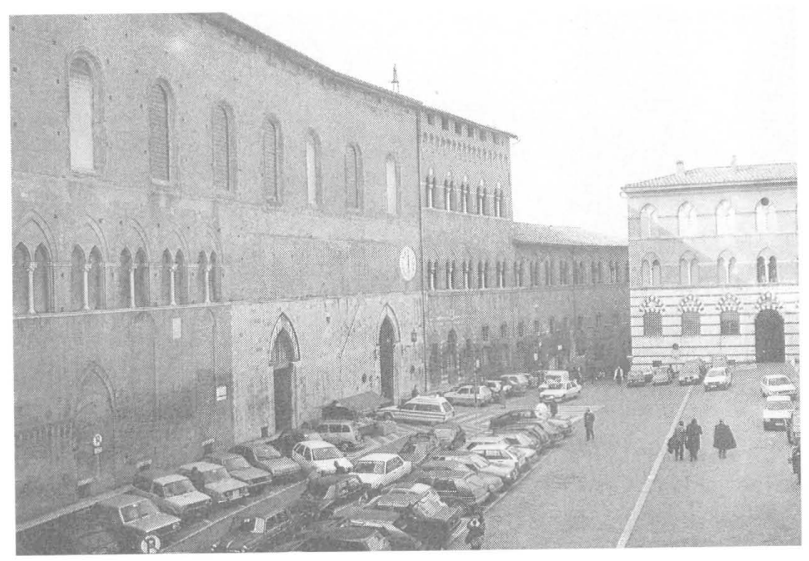

Fig.2.- A la izquierda, la fachada del Spedale di Santa Maria della Scala, en la plaza del Duomo de Siena, una arquitectura monumental que sigue cumpliendo su función desde el s. YIII.

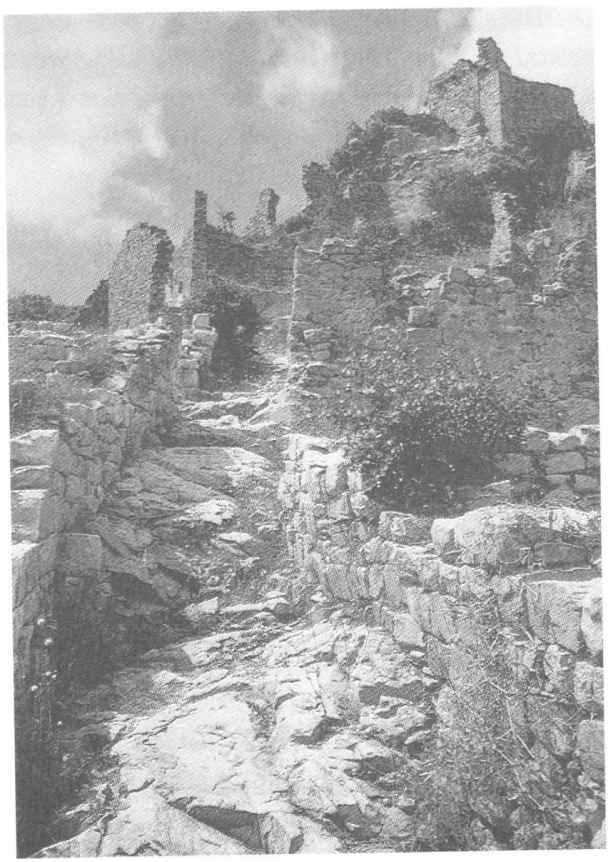

Fig.3.- Un callejon del pueblo medieval de Rocca San Silvestro (prov. de Livorno), abandonado durante el siglo XIV y objeto de una vasta campaña de excavaciones desde 1984.

(c) Consejo Superior de Investigaciones Científicas objetivos posible ${ }^{2}$-del mismo modo que se comenzaba a hacer con la cerámica medieval- abría una puerta que la curiosidad intelectual de la joven disciplina y la recién nacida revista del mismo nombre-Archeologia Medievale-, no podían dejar que se les escapara (Figs. 1, 2 y 3). Era inevitable que el desarrollo de una arqueología medieval o mejor dicho, postclásica- la llevase a un enfrentamiento con la historia de la arquitectura y con los problemas relacionados con la conservación de obras arquitectónicas: los aspectos funcionales, las modificaciones de uso, las transformaciones de la arquitectura, son el pan de cada día del arqueólogo que interviene en los centros urbanos, en estructuras monumentales o en aldehuelas abandonadas ${ }^{3}$. La mayor atención con que se observaron las obras de fábrica, con la vista puesta en reconocer los cambios menos visibles, las dimensiones de los elementos, las huellas de diversas herramientas, la calidad de los morteros, tuvo como resultado casi inmediato la constatación de que una fábrica es casi siempre el producto de una serie de actuaciones constructivas que se suceden en el tiempo (Figs. 4 y 5). El siguiente paso, la experimentación con un instrumento adaptado al registro y transmisión de todo lo observado, fue casi automático: en los mismos años se estaba difundiendo en la arqueología italiana, a través del trabajo de Andrea Carandini, el instrumento estratigráfico, y qué mejor ocasión se podía presentar para experimentarlo en otros campos que respondian a las mismas leyes de formación pero que hasta entoncês se habían estudiado con otros instrumentos ${ }^{4}$.

$\mathrm{El}$ acento puesto con el paso del tiempo ha hecho necesario distinguir expresamente indicadores cronológicos, entre los que se encuentran todas las fuentes tradicionales, junto a instrumentos específicos ${ }^{5}$ desarrollados en el curso de la investigación y que, en la óptica de una utilización análoga a las prácticas llevadas a cabo en los estratos arqueológicos horizontales, permiten ampliar el conocimiento del contexto, que por analogía podemos llamar de la estratigrafía vertical: las técnicas constructivas, los motivos decorativos, el acabado de los elementos, la factura de los huecos, etcétera.

\section{La estrategia}

En la cadena ininterrumpida de materias que tiene ante sí el estudio de la arquitectura, que van desde el conocimiento de la historia del asentamiento hasta la composición de las argamasas -aunque la cadena podría alargarse hacia lo infinitamente pequeño o hacia problemas aún más generales-, el concepto de escala asume un valor particular, ya que, al ser la cadena prácticamente infinita, debemos escoger y estudiar sólo una parte de ella. Por ello ampliamos algunos eslabones del sector e introducimos subdivisiones, quizás arbitrariamente. Se trata, en lo esencial, de definir una especie de escala conectada a una unidad de referencia, con grados de registro y documentación diversificados en la 
investigación -de lo general a lo particular-, con la que relacionar la estrategia más adecuada, pero adoptando el mismo método de análisis: la lectura estratigráfica. Esta operación parece metodológicamente correcta, ya que al pasar de un eslabón a otro, se da solamente un salto de escala.

En Italia, la posibilidad de referirse a niveles de profundización diferentes se ha afrontado de dos maneras distintas. La primera nace de la experiencia sobre el terreno, llevada hasta el aspecto concreto de la subdivisión por niveles de profundización en el estudio estratigráfico de la edificación, y ha sido enunciada por Brogiolo 6 . La otra surge por la exigencia de reunir en una estructura funcional más amplia, que pueda cubrir todo el campo de actividad arqueológica, las numerosas fichas presentadas en Italia por el Instituto Central del Catálogo y Documentación (ICCD). Mientras Brogiolo distingue cinco niveles en los que confluyen los datos relativos a los Complejos Arquitectónicos (CA; en italiano Complessi Architectonici, $\mathrm{CA}$ ), Cuerpos de Fábrica (CF; Corpi di Fabbrica, CF), Alzados Generales (AG; Prospetti Generali, PG), Alzados de Detalle (AD; Prospetti Particolari, PP), Unidades Funcionales o Ambientes (UF; Unità Funzionali, Ambienti, UF), etc. , hastallegar a las Unidades Estratigráficas Murarias (UEM; Unità Stratigrafiche Murarie, USM); nosotros adoptamos tres niveles? . El primer nivel, más rápido y sumario, lo limitaremos a la determinación del contorno de las grandes masas de obra, a los entramados constructivos y a los diferentes materiales, y ha de registrarse sobre una base planimétrica. El segundo nivel se ocupará de reflejar en un fotoplano o un relieve gráfico (Fig. 6), aunque sea aproximado y sin demasiados detalles, los cambios de cota del suelo -legibles mediante el desplazamiento de los huecos-, el contorno de las UEM -individualizadas por la diferencia de aparejos y de las dimensiones macroscópicas de los materiales, y por la variación de las formas decorativas, las dimensiones de los huecos, los elementos ornamentales si los hay o de una determinada factura, etc.acompañando el registro gráfico (Fig. 7) con la redacción de la ficha correspondiente. Finalmente, en un nivel más detallado, tendremos el registro analítico de todas las UEM que se puedan distinguir, caracterizadas por sus componentes constructivos singulares-de los que tendremos presente las huellas de su ejecución y el acabado de sus superficies-, el tipo de aglomerante, el espesor de las juntas y, si se trata de obra de ladrillo, las medidas al milímetro de un número de elementos estadísticamente significativo. En este último caso, el registro gráfico debe ser lo más completo posible -compatible con las exigencias del proyecto-, aunque puede considerarse útil, como representativo de un conjunto más amplio y homogéneo, el dibujo de una parte-alrededor de $100 \times 150 \mathrm{~cm}$ - de la técnica constructiva (Fig. 8).

Pero también se puede relacionar la escala jerárquica de niveles de profundización con todo el complejo constructivo,

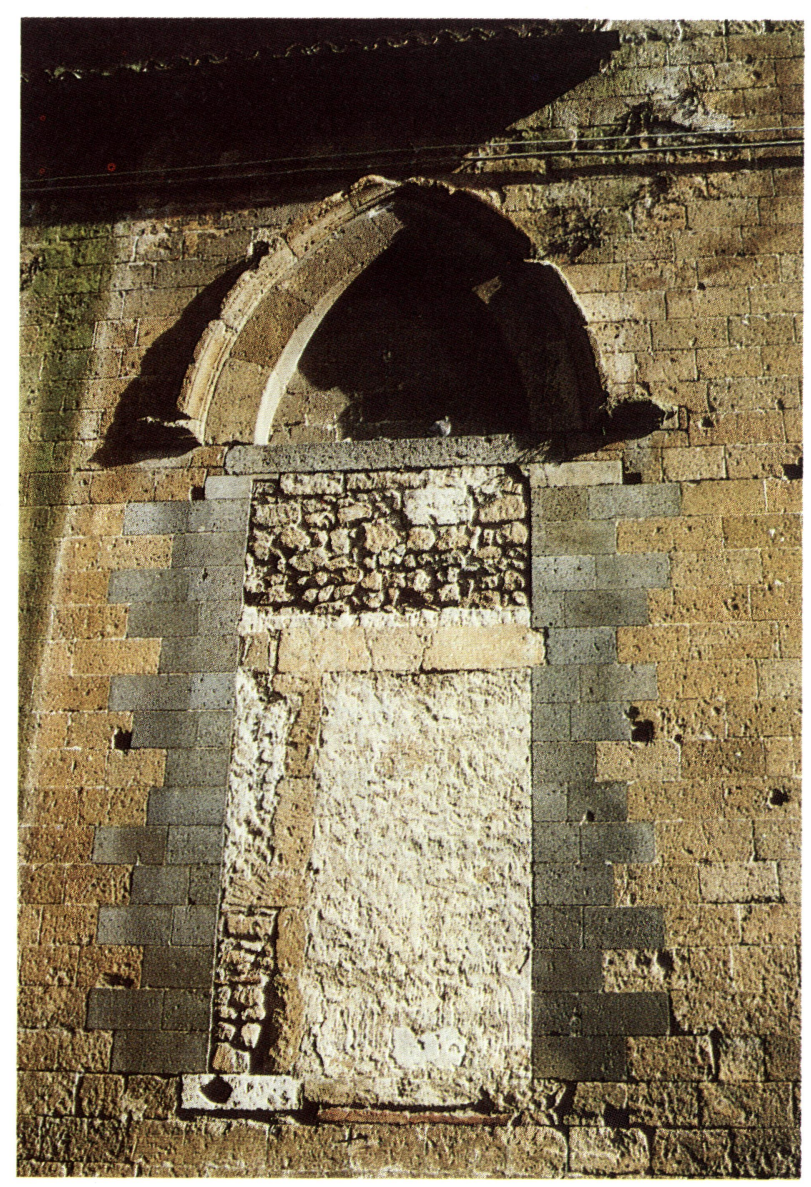

Fig.4.- Las vicisitudes constructivas de una ventana de la iglesia de San Francesco de Orvieto (prov. de Terni), son puestas en evidencia por el empleo de diversos materiales constructivos.

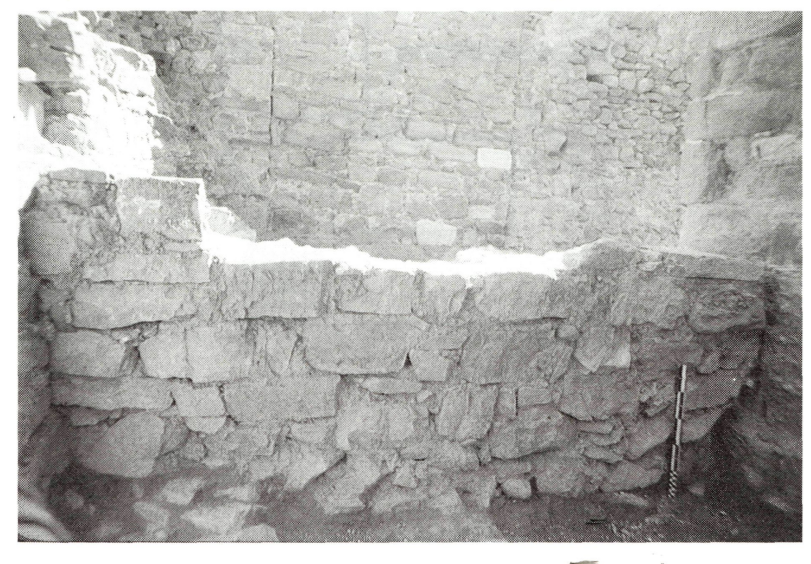

Fig.5.-En un castillo de la costa toscana, Scarlino (prov. de Grosseto), la excavación arqueológica ha evidenciado numerosas fases constructivas y transformaciones de la arquitectura. 


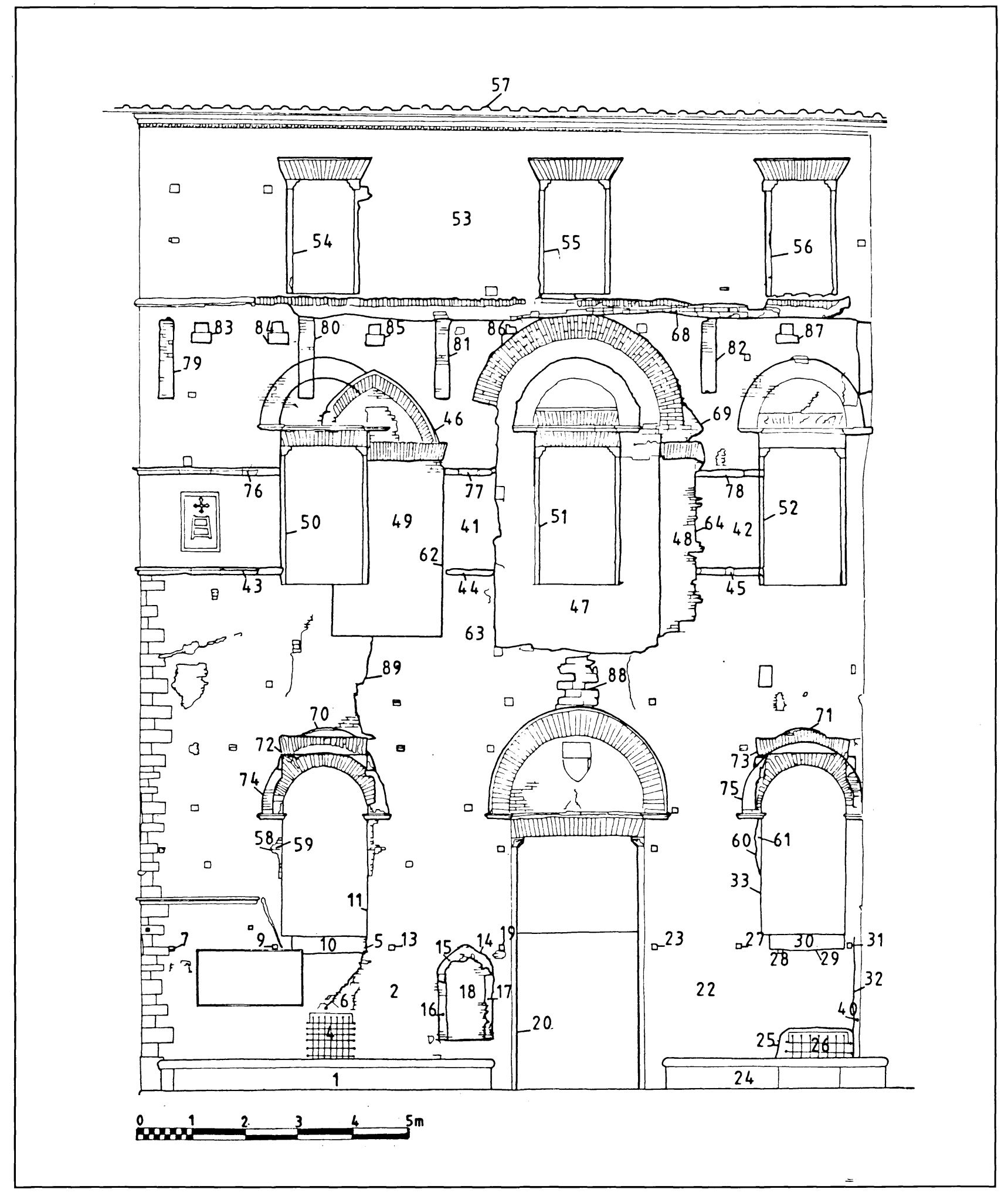

Fig.6. - El levantamiento fotogramétrico que ha servido de base para el registro de las transformaciones de la fachada del Convento delle donne, del s. $X I V$, en el Spedale di Santa Maria della Scala, en Siena. 


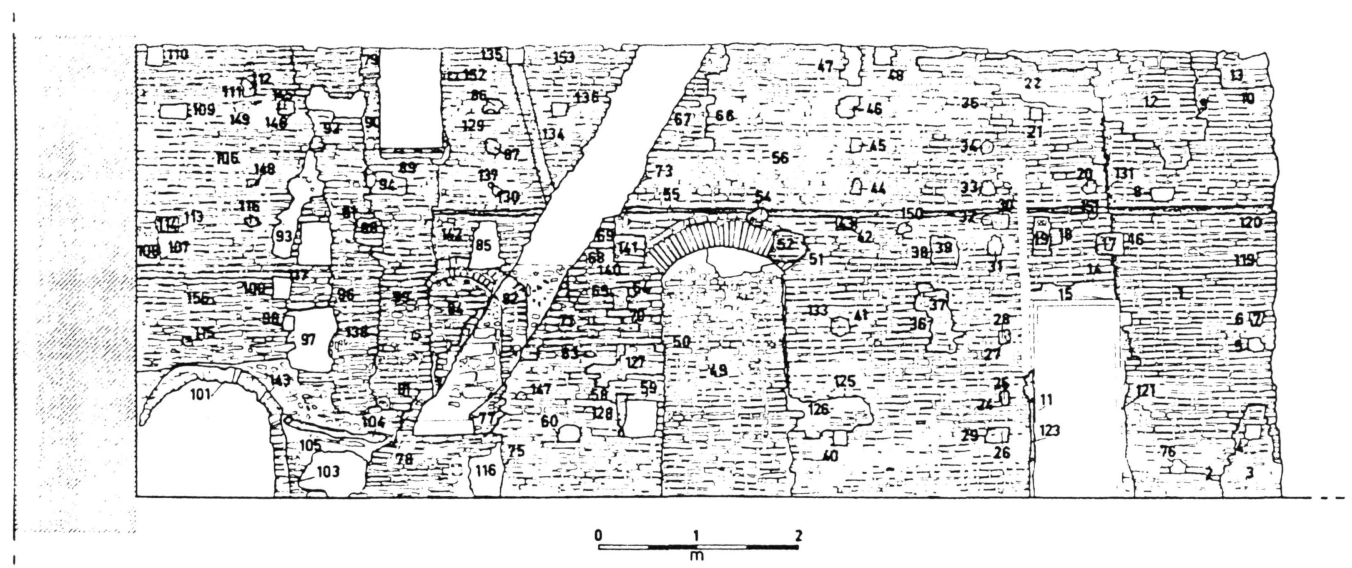

Fig.7.- Levantamiento realizado para evidenciar los episodios constructivos del muro que sustenta el fresco de la Maestà realizado en 1315 por Simone Martini- y facilitar la identificación de las causas de su degradación. Siena, Palacio Municipal.

subdivididoen partes más fáciles de estudiar. En la situación que representa mejor el conjunto del producto edificado -el centrourbano- por los criterios que rigen las intervenciones, junto a la escala es esencial definir el modo de delimitar los campos de observación. Una fase de compresión o de reducción que se hace necesaria en vista de la amplitud del área estudiada que, generalmente, parece excluir una intervención de tipo analítico.

Se trata, en esencia, de pasar de las informaciones de carácter cuantitativo -que responden a las preguntas de ¿cuántos y dónde están?, típicas del arqueólogo- al valor cualitativo basado en la evidencia documental - ¿cómo son?-, mediante operaciones de urban fieldwork, o sea de análisis del tejido urbano y de la estructura que permanece en pie. Todavía dentro de la fase cuantitativa, procederemos de un proceso extensivo a otro intensivo de la investigación, privilegiando la base planimétrica cuando se analizan partes amplias de lo edificado y dando más importancia a los alzados cuando deban comprender los pasos constructivos, las transformaciones en el tiempo de un edificio en particular o de pequeños cuerpos de fábrica.

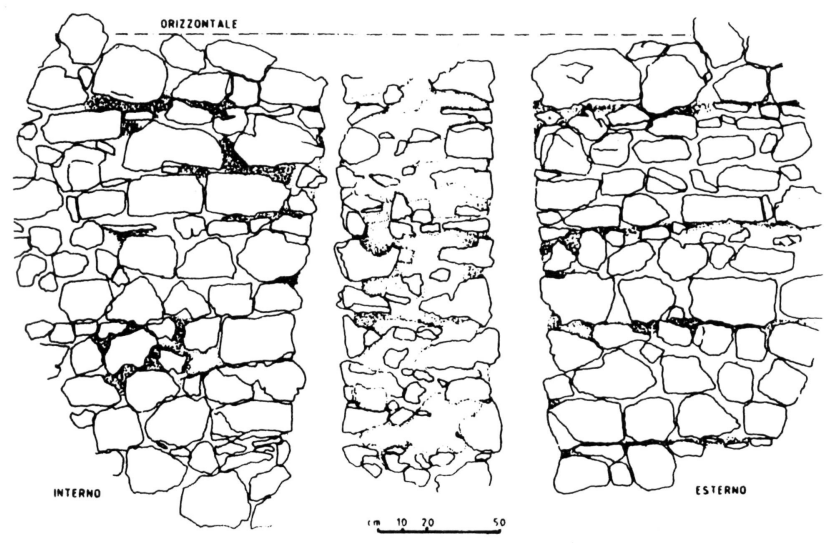

Fig.8.- El registro gráfico por "muestreo(o catas)" de la técnica constructiva: una fábrica medieval de la ciudadela del castillo de Montarrenti(prov. de Siena).
Este modo de descomponer el tejido urbano edificado tiene precedentes y un paralelismo estrecho, por un lado, con la estrategia seguida en algunos Planes Reguladores de los centros históricos italianos, que distinguen un edificio aislado, o grupos de edificios homogéneos, para fundamentar las prescripciones de carácter urbanístico, con indicaciones, principalmente, sobre los criterios que han de adoptarse para la restauración y conservación de los edificios; y por otro, con la estrategia seguida en un terreno más propio de la arqueología como es el de la prospección de superficie.

Proponer una estrategia idéntica para el estudio del territorio y para el análisis de un centro urbano es sólo una contradicción aparente. Es cierto que las situaciones parecen completamente distintas: en el centro urbano la superficie es reducida, pero, con su más densa serie de informaciones, la diacronía está casi siempre más comprimida, los materiales y los fósiles-guía son variados -técnicas constructivas en vez de acervo mobiliario-, es diferente la escala del modelo de asentamiento-edificios en vez de núcleos de habitación- y además difiere notablemente de la estrategia adoptada para las excavaciones, dondeel aspecto destructivo de la operación permite pocas alternativas a la exigencia de un registro global o, al menos, que sea compatible con los recursos del proyecto. Pero permanece constante la sistemática del análisis y la técnica de registro. Pasar de la identificación de cada Unidad Estratigráfica Muraria singular-el estudio de las técnicas constructivas- a un conjunto más amplio, al conjunto de la actividad edificadora constituida por los Cuerpos de Fábrica -los edificios-, significa considerar cada CF como fábricas cronológicamente unitarias que representan una etapa del proceso formativo de la aglomeración urbana ${ }^{8}$,y, por lo tanto, estratos a todos los efectos (Figs. 9, 10 y 11). Y es una operación que, con la ayuda determinante del conocimiento de las técnicas constructivas y de la identificación de las relaciones estratigráficas, exige un tiempo relativamente corto, en 


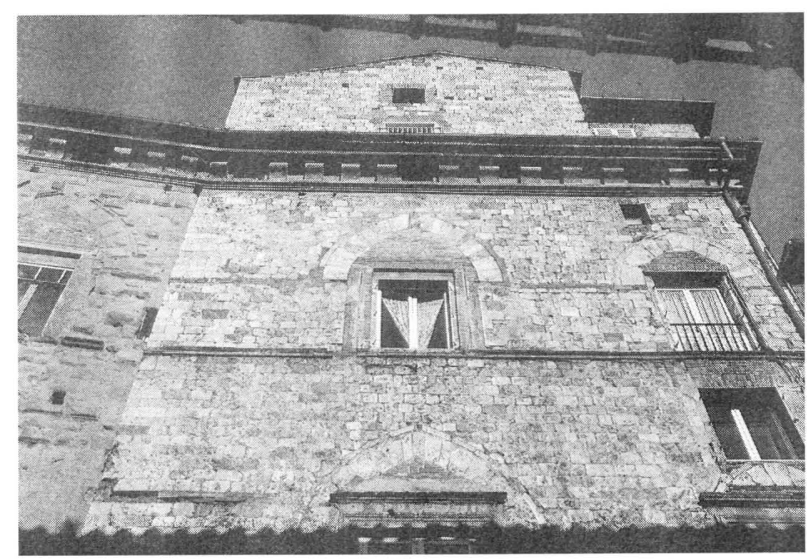

Fig.9.- La presencia de entramados constructivos permite distinguir la extensión original de un edificio. La torre de los Forteguerri en Siena, muestra una diferencia de los materiales de construcción.

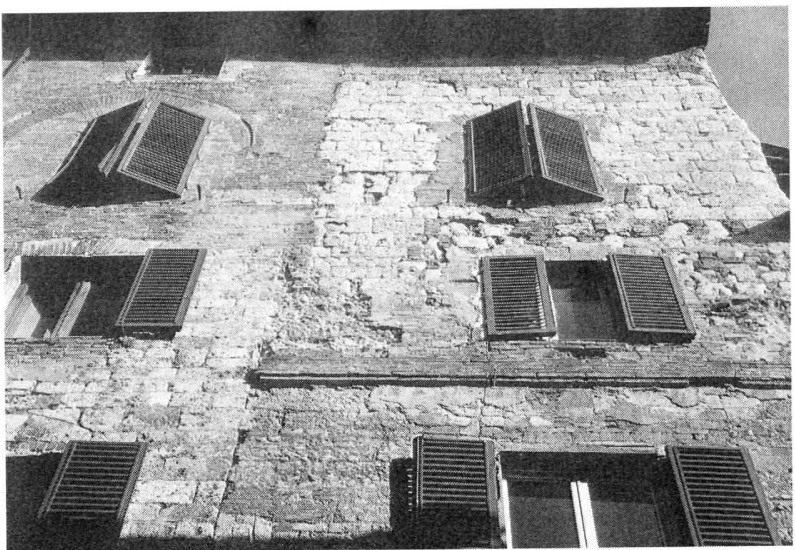

Fig.10.- Acontecimientos constructivos más complejos -referidos a más fases constructivas- se reconocen en el examen de los entramados de la torre de los Ugurgeri de Siena.

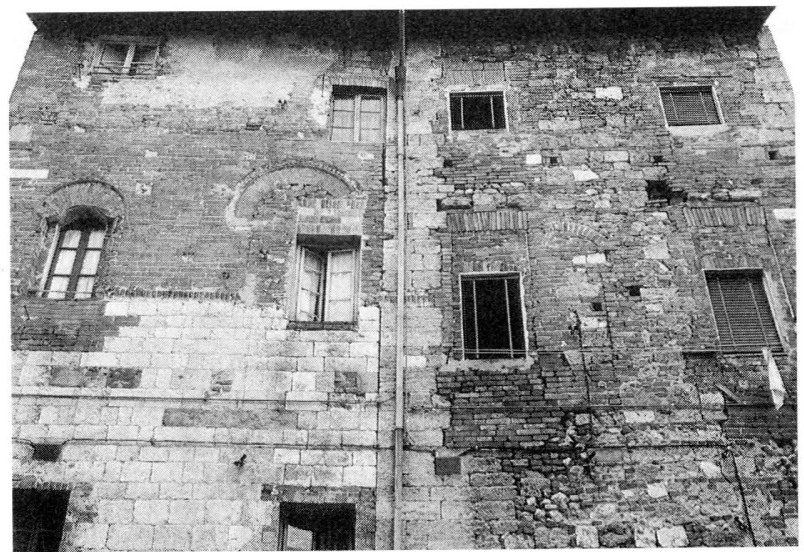

Fig.11.- También la construcción menor del centro de Colle Valdesa (prov. de Siena), permite reconocer correctamente la extensión original y la evolución posterior de la construcción. especial en medios urbanos donde sea posible observar directamente el soporte murario. Amén de que la exigencia de documentación requerida por las ' rápidas transformaciones de un centro urbano moderno, impone hacer una selección urgente y esta selección será tanto más incisiva cuanto mayor sea el grado de conocimiento de las vicisitudes constructivas del asentamiento. Continuando por el encuadre ideal de la escala de las intervenciones, cuando pasemos a analizar los edificios, tendremos individualizaciones de grandes masas de obra y sus relaciones estratigráficas recíprocas dentro de los Alzados Generales que constituyen los Cuerpos de Fábrica o, si se trabaja en el tramo más analítico de la escala, la diversidad de aparejos, de dimensiones y de ejecución de los materiales empleados en el mismo Alzado de Detalle, hasta llegar a las dimensiones de los ladrillos y a la composición de la argamasa dentro de la misma UEM.

En el estado actual del conocimiento, el punto nodal de la estrategia de intervención en el estudio del depósito vertical es la determinación de cada acción constructiva-y también de demolición-, la UEM. La UEM puede ser considerada, por un lado, como un conjunto de partículas elementales los materiales de construcción- $y$, por el otro, como parte de una estructura más compleja -el edificio- (Figs. 12, 13 y 14). Con esta dualidad, es posible colocar dentro de un esquemageneral más articuladoinclusola rigurosa analítica de una lectura de la edificación desarrollada para cada una de las acciones constructivas, a la vez que se podría dar una mayor importancia a los análisis naturalistas que se realicen sobre los materiales de construcción, de manera cada vez más profunda, si introducimos, en un contexto en el que se conozca la historia constructiva de la estructura, el sitio donde se haya efectuado el muestreo. Y esto vale también en el caso de las consideraciones sobre la estática del edificio, que, a menudo, viene modelizado como una serie de cuerpos homogéneos, sin tener en cuenta las modificaciones que se han sucedido en el tiempo.

\section{La práctica operativa}

Afrontar un aspecto material y concreto, en este caso la misma edificación en todos sus aspectos característicos, permite separar el momento de registro de las evidencias del de la interpretación que se pueda extraer de estas evidencias. La misma atención dedicada a definir los contornos del contexto homogéneo -por los momentos constructivos, los materiales empleados, las técnicas utilizadas, etc.- nos ha permitido intentar entender los criterios seguidos por los constructores al realizar la obra. Observación que no supone un mero ejercicio intelectual de catalogar una serie de tipos, sino que constituye una verificación fundamental de los modos $\overline{\bar{y}}$ los tiempos con que se ha formado el contexto y de Tas posibilidades informativas que se han dado recíprocamente entre historia 


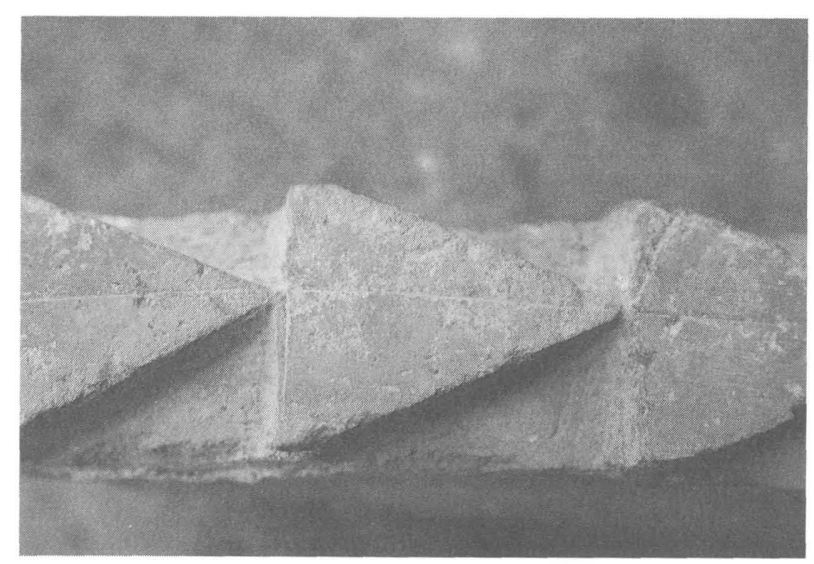

Fig.12.- El acabado de la superficie de un ladrillo de mediados del s. XII caracteriza la tecnología utilizada para preparar la decoración.

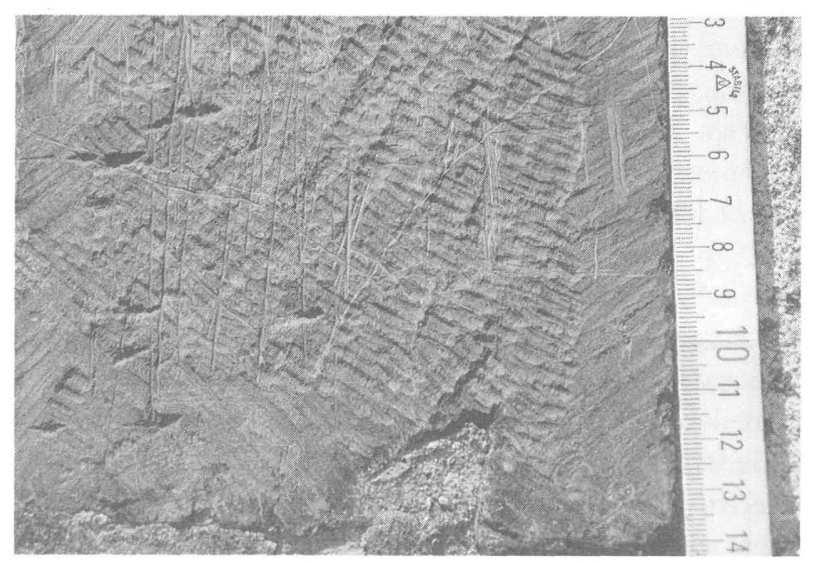

Fig.13.-Las huellas de una herramienta de dientes cuadrados, "gradina", en la piedra de un edificio religioso medieval.

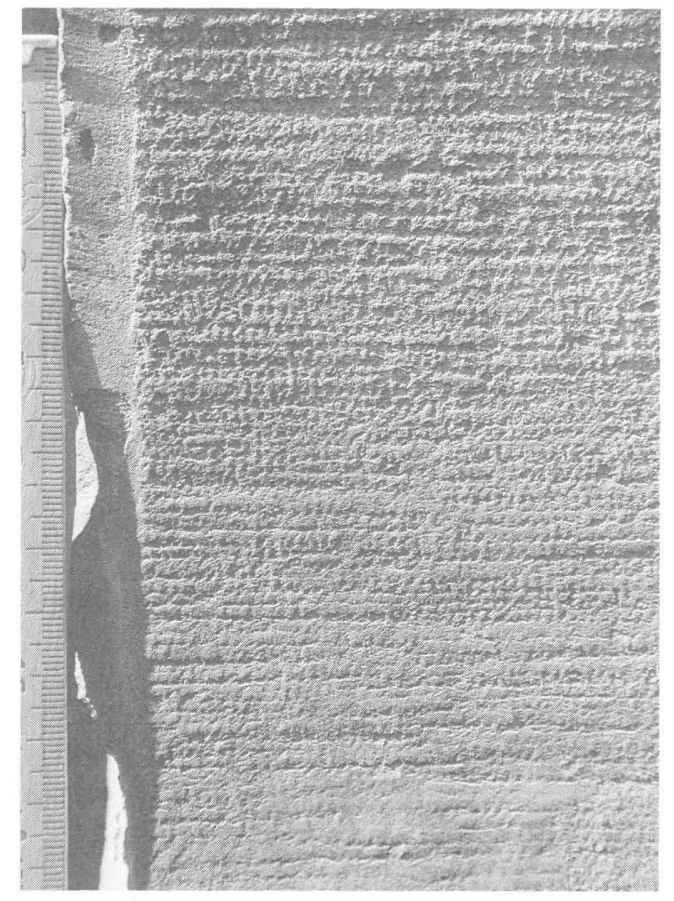

Fig.14.- La huella de una "martellina", de dientes de "grano de cebada", en la moldura de una ventana del s. XVI. social, técnica constructiva, fuentes escritas, epígrafes y arquitectura.

Para el registro se prevé la redacción de una ficha operación absolutamente necesaria para describir cada unidad estratigráfica individualizada-, con campos preestablecidos y constantes, que aseguran una respuesta a las preguntas más significativas, eliminando así la posibilidad de recoger la información de modo incompleto y arbitrario. El registrográfico de las evidencias materiales, el dibujo de la unidad, debe considerarse parte integrante de la documentación escrita. El registro es una operación dirigida a identificar las acciones físicas -que se pueden tocar y observar y por ello son mensurables- que se insertan . sucesivamente en diagramas globales, donde se deja poco lugar a impresiones subjetivas. Se trata de individualizar los contornos de todas las acciones constructivas homogéneas y las relaciones del tipo antes de, después de, contemporáneo $a$, entre cada acción -estratos o cuerpos de fábrica-. En la elaboración del diagrama se condensan las relaciones temporales relativas que se han reconocido y registrado entre cada acción constructiva y sólo en un momento posterior se interviene interpretando, de acuerdo con los conocimientos propios del estado de la investigación y la capacidad subjetivaindividual, lafunción y la cronología absoluta de las acciones incluidas en el diagrama. Está ya comprobado que en la actividad de una obra constructiva algunos momentos se apartan del trabajo rutinarioy pueden dejar trazas específicas. Cuantos más sean esos momentos, más fácil resultará la interpretación. La presencia de mechinales para el andamiaje, únicamente en ciertas posiciones, puede señalar una actividad que merece mayor atención (Figs. 15 y 16), la existencia de elementos con motivos decorativos -huecos, coronamientos, etc.-, son aspectos que transcienden la actividad normal e introducen un valor de excepcionalidad en el proceso constructivo. Estas trazas se encuentran siempre en las obras y es oportuno interpretar su significado, teniendo presente que en ocasiones no es fácil llegar a entenderlas debidamente. Hay también situaciones en las que la tradición enraizada puede inducir conclusiones, siendo entonces necesario despojarse de las nociones preestablecidas y registrar sólo la evidencia material.

En este segundo paso se pueden correr riesgos de misunderstanding, pero están previstos $\mathrm{y}$, sea como fuere, no podrán modificar las relaciones generales del diagrama.

Para definir de manera más exacta las propias zonas de intervención, parece que el mayor esfuerzo se debe dirigir a identificar los resultados de las actuaciones constructivas positivas -el perímetro de las ampliaciones, las intervenciones de restauración, de apuntalamientos, las reintegraciones, etc.- y de las negativas -la superficie residual de las extracciones o derrumbes de partes construidas, de la evolución de los daños, etc.-. Estas operaciones deben hacerse sobre el terreno, en contacto 


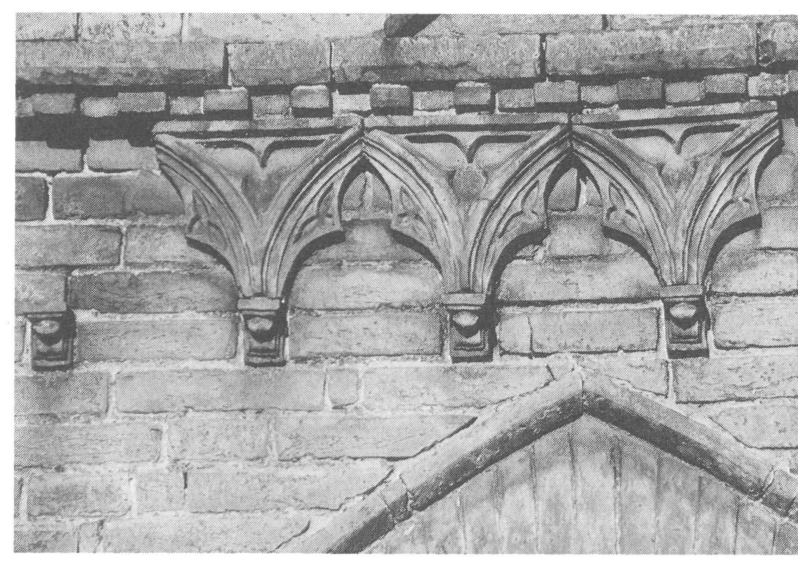

Fig.15.- La técnica de montaje de la ornamentacion de ladrillo de un palacio del s. XV en Siena.

directo con el edificio -del mismo modo que el arqueólogo no puede delegar en otros las operaciones de excavacióndonde la experiencia y el conocimiento de los problemas desempeñan un papel absolutamente prioritario. Sólo en la fase de interpretación, con el fin de llegar a un resultado más exhaustivo, se podrá buscar el concurso de otras especialidades.

\section{4. ¿Cómo se utiliza la información?}

Si podemos reconstruir los episodios constructivos de la arquitectura, la microhistoria de uno o más complejos edificados, los resultados se podrán utilizar en distintas direcciones. Hasta ahora, los argumentos de carácter histórico, enfrentados a la investigación arqueológica, han sido esencialmente dos: la caracterización de la técnica constructiva ${ }^{9}$ y la identificación del modelo constructivo de referencia en las distintas épocas históricas ${ }^{10}$. Pero incluso la capacidad de vincular las técnicas constructivas a una cronología absoluta nos permite ampliar también el campo de operaciones a la determinación de las fases del desarrollo constructivo de todo un centro habitado ${ }^{11}$, cuando el teatro de la observación está constituido por el tejido edificado entendido en su totalidad o, viceversa, contextualizar el detalle de daños más o menos evidentes. En este campo las investigaciones realizadas, todavía limitadas en cuanto a su número y en cuanto a su importancia geográfica, son particularmente interesantes, sobre todo por la puesta a punto de criterios de descodificación de las posibilidades informativas contenidas en la propia obra, más aún que por los resultados obtenidos.

Lo que sigue representa una reseña de las posibles utilizaciones.

\section{A) La caracterización de las técnicas constructivas.}

La atención dedicada -y no podía ser de otro modo- a algunas características propias de las técnicas constructivas

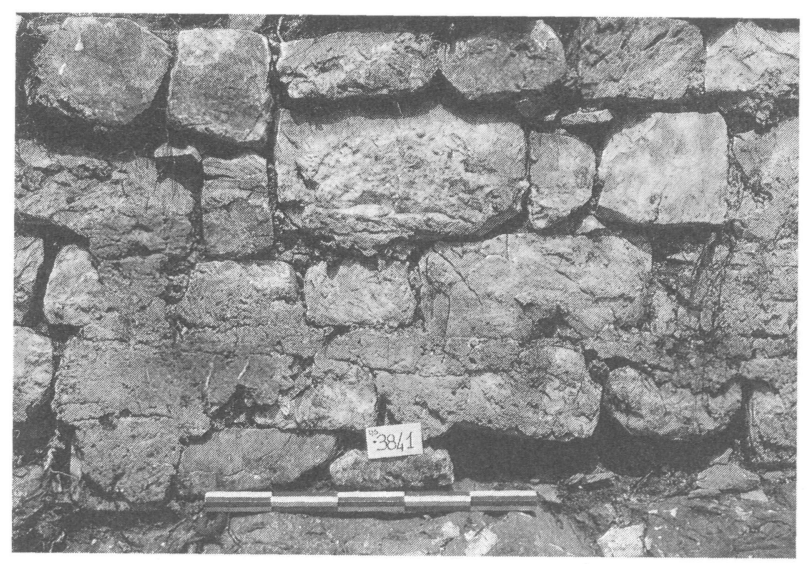

Fig. 16. - L na fabrica de piedra enlucida del s. Xll, que ha permanecido oculta desde mediados del s. XIII

-a nivel de análisis, materiales, aparejo y secciones, elaboración y acabado, argamasa-, siempre comparables y reagrupables en Atlas de zonas geológicas homogéneas, nos muestra diferencias propias de las épocas históricas y de los contextos socio-económicos en que se insertan, pero también técnicas comunes a materiales y contextos regionales diferenciados. Salvo pequeñas ampliaciones del campo de intervención, todavía son válidos los puntos propuestos por primera vez en 1987 en Bressanone ${ }^{12}$. Pero se pueden hacer desarrollos interesantísimos realizando un verdadero salto de escala, profundizando ya sea en el estudio de las dimensiones de los ladrillos -la mensiocronología, esto es, la posibilidad de relacionar la dimensión de los ladrillos con las distintas épocas históricas ${ }^{13}$ - obien en la definición de la técnica constructiva de los huecos -la cronotipología, la caracterización de las técnicas constructivas de los huecos en distintos momentos históricos ${ }^{14}$.

La continua comparación y convergencia de los datos que surgen de los objetivos de la investigación, permiten la construcción muy esquemática de modelos, hoy en día forzosamente parciales, pero no por eso menos comprometidos. En Italia, entre los siglos IX y XVI -pero incluso más allá- los puntos clave que merecen sin duda una atención especial -y la concentración del interés cuando nos interesa comprender el desarrollo de la técnica constructiva local- parecen ser los siguientes:

I-El momento del paso de los entramados de madera-u otra estructura más delicada realizada con material perecederoa una estructura de fábrica;

II -verificar la dispersión de la distribución geográfica y la cronología del desarrollo de las técnicas de construcción que utilizan sillares bien escuadrados y alisados -la construcción románica para simplificar-;

III -la introducción -o el uso generalizado- del ladrillo en la construcción -en interesante relación con una necesidad de edificación en rapidísima expansión-; 
IV -el abandono de paramentos con hiladas paralelas horizontales, en algunas zonas ya desde la primera mitad del s. XIV, cuando se adopta el estilo constructivo llamado a la moderna ${ }^{15}$.

Responder a estas preguntas significa buscar un hilo conductor, una línea que permita situar todas y cada una de las características en un esquema general y que nos ayude a comparar los datos de las investigaciones, $y$, al mismo tiempo, dé claridad en las operaciones de lectura de la estratigrafía de la edificación, como sucedió con la renovación del estudio de la cerámica postclásica, con el fin de elaborar Atlas subregionales de las técnicas constructivas tradicionales. Estos mismos Atlas parecen muy interesantes para los arquitectos que propugnan la vuelta a las técnicas constructivas tradicionales, más compatibles con el entorno edificado existente, e incluso en las intervenciones de adecuación antisísmica ${ }^{16}$. En efecto, recientes episodios trágicos han renovadoel interés por comprobar la resistencia mecánica de las construcciones tradicionales.

\section{B) La definición del modelo constructivo.}

La reconstrucción de la microhistoria del edificio, desde la etapa de cimentación a las transformaciones y las restauraciones más recientes, es el paso indispensable para definir los modelos de la construcción. La aplicación del método se experimentó primero sobre los restos de las habitaciones encontradas en excavaciones, luegoen edificios aún en uso y, posteriormente, en los grandes complejos monumentales, donde una mayor cantidad de fuentes documentales ha permitido una comprobación cruzada sumamente convincente de las actividades constructivas $a$ la vista respecto a la fuente material ${ }^{17}$.

La investigación sobre el terreno ha demostrado lo difícil que resulta sostener la suposición de un tipo de edificio repetible. Se debe obrar con extrema cautela cuando se tienen que identificar los caracteres distributivos, las subdivisiones funcionales, las soluciones técnicas y formales que deberían constituir el modelo paradigmático de la construcción de determinados períodos históricos. A menudo se pretende reconocer un tipo de construcción basándose en exámenes -al menos formalmentedimensionales y/o distributivos, pero una observación más cuidadosa revela que, frecuentemente, el tipo resulta homogéneo sólo en lo que respecta a la estructura de la propiedad. La misma situación se presenta incluso en situaciones en las que el contexto urbano pudiera parecer más regular, como es el caso de las terre nuove florentinas ${ }^{18}$ (Figs. 17, 18, 19 y 20).

La microhistoria del edificio resulta el eslabón de enlace con la primera fase del análisis -en las operaciones de restauración arquitectónica- y con las investigaciones del comportamiento estático de todo el conjunto de la obra. En las últimas experiencias estamos de hecho comenzando a (c) Consejo Superior de Investigaciones Científicas Licencia Creative Commons 3.0 España (by-nc)
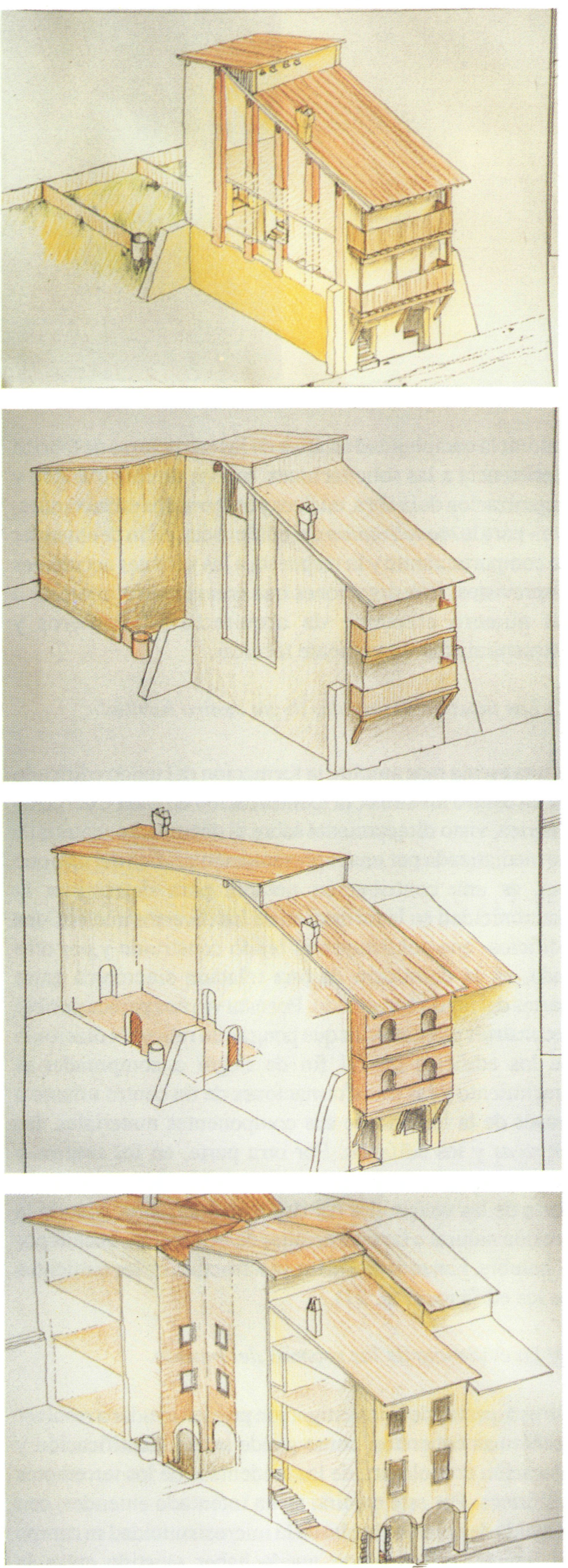

Figs. 17,18,19 y 20.- Las principales fases constructivas de un edificio en hilera de la "terra nuova" florentina de San Giovanni Valdarno: 17, en la segunda mitad del s. XIV; 18, durante el s. XV; 19, entre fines del s. XVy el s. XVII; 20, desde el s. XVIII. 


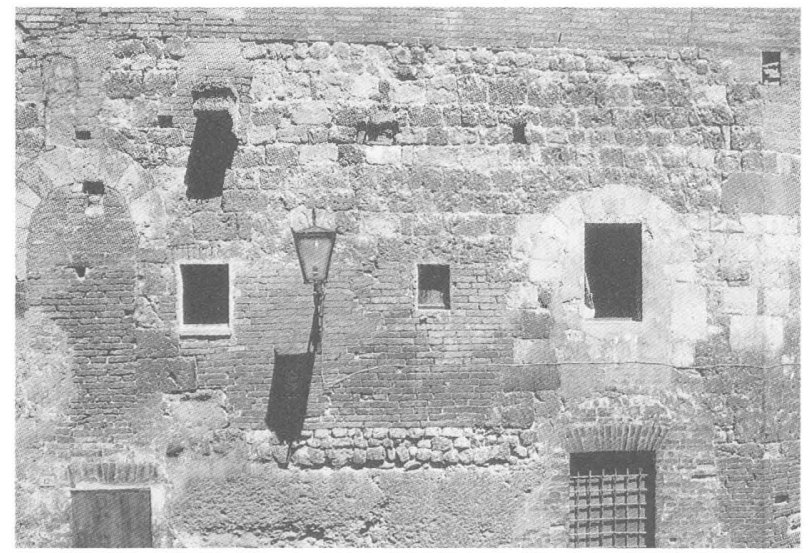

Fig.21.- Los cimientos -las tres hiladas de sillares almohadilladossobre los estratos geológicos -arenas y gravas del plioceno-, puestos a la luz por el hundimiento de la superficie exterior, probablemente en la primera mitad del s. XIV.

valorar la complejidad estática de las estructuras de fábrica -resistencia a las solicitaciones, modos de construcción y organización de la obra, núcleos y entramados estructurales, etc.- para la modelización del edificio con el fin de entender su comportamiento y la respuesta a los posibles accidentes imprevistos, investigaciones que comienzan a interesar a un número creciente de arquitectos, ingenieros y estructuralistas -la vertiente técnica-.

\section{C) Las fases de desarrollo de un centro habitado.}

A una escala más amplia, la formación del tejido edifícado de un centro histórico, la identificación de cada Cuerpo de Fábrica, visto directamente sobre el documento material y no mediatizada por una representación gráfica del tipo que sea, es una contribución notable para determinar la diacronicidad en la formación de los diversos núcleos -los edificios- que constituyen el tejido construido y,por otro lado, en la definición de una relación sincrónica entre partes del tejido construido. Por esta vía nos parece posible reconstruir planimetrías que pongan en relieve la diacronía de los edificios con el fin de llegar a comprender el crecimiento y las transformaciones de un centro urbano a través de la historia de sus componentes materiales: las fábricas y los edificios. Por otra parte, en los centros y pueblos de altura, no parece imposible determinar una parte de las vicisitudes del depósito horizontal cuando la erosión natural o las operaciones de extracción, hechas por el hombre, han sacado a la luz la estructura de los cimientos de los edificios (Fig. 21).

\section{D) La evidencia de los seísmos del pasado.}

Otros aspectos de la investigación pueden ayudar a resolver problemas concretos, como puede ser la identificación y ubicación cronológica de las evidencias de los terremotos históricos. Por este camino se ha intentado entender, con la ayuda de quien ha hecho de la microsismicidad su campo de interés, qué relacion puede haber existido entre la intensidad del seísmo y las características constructivas de los edificios, la calidad de la obra -en la escala MCS, la intensidad alcanzada por el epicentro del terremoto se determina refiriéndolo al grado de destrucción sufrido, especialmente por los edificios- y una primera asignación cronológica de los daños y los trabajos de reconstrucción de la obra, atribuibles a una actividad histórica atestiguada o no por las fuentes escritas ${ }^{19}$ (Fig. 22).

En resumen, para concluir, en la arquitectura disponemos de un enorme instrumento que registra fielmente una grandísima cantidad de sucesos, ya sean hechos humanos o naturales; por ahora hemos creado una técnica de descodificación que permite leer una parte de tal registro, pero la riqueza de datos y la fidelidad de las restituciones son tentadoras $\mathrm{y}$, a pesar de la prudencia que requiere la investigación, no nos parece fuera de lugar apostar, en el análisis de las obras, por un futuro cada vez más satisfactorio $\mathrm{y}$ variado en este sector de la investigación.

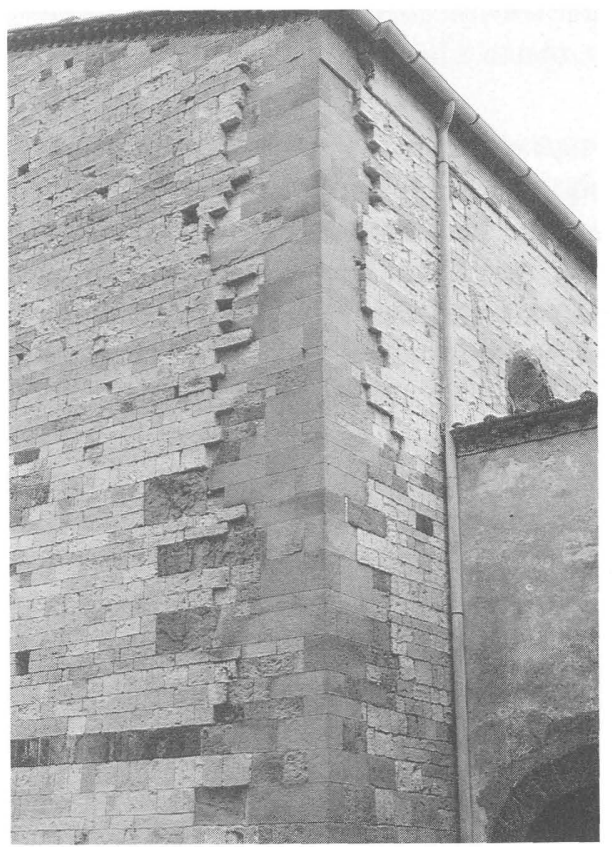

Fig.22.- La recuperación de la continuidad estructural de la esquina de un edificio religioso: Sant' Agata di Scarperia (prov. de Florencia), realizada con operaciones de "cosido y descosido", la primera vez tras el terremoto de 1542 y la segunda después de 1920. 
${ }^{1}$ Una historia reciente del nacimiento y desarrollo del análisis estratigráfico en Italia se encuentra en R. Tagliabue, Architetto e archeologo. Confronto fra campi disciplinari, Milán 1993.

${ }^{2}$ T. Mannoni, L 'analisi delle technice murarie in Liguria, en Atti del colloquio internazionale diarcheologia medievale, PalermoErice, 1974. Palermo 1974, pp. 291-300.

${ }^{3}$ Un testimonio de la necesidad de establecer relaciones entre el arquitecto y el arqueólogo fue el congreso Archeologia $e$ pianificazione dei centri urbani, celebrado en 1978; cfr. el número monográfico ArchMed, VI (1979).

${ }^{4}$ Una prueba de la racionalidad del instrumento estratigráfico lo tenemos en su larga tradición de aplicación, aunque intermitente y sin conexiones académicas. El estudio de Van Deman sobre el Atrio de Vesta en Roma, cfr. E. B. Van Deman, The Atrium Vestae, Washington 1909, seenmarca en la fértil etapa positivista de comienzos de siglo que, como G. Boni, hace uso de observaciones que podemos definir como protoestratigráficas. Desde entonces tenemos numerosos trabajos, aunque dispersos en publicaciones no orgánicas, caracterizados por una atenta observación de las estructuras materiales. Entre otros me place recordar algunos estudios de $\mathrm{M}$. Pera sobre la arquitectura medieval pisana, $c f r$. M. Pera, La zona di S. Pietro in Vincoli in Pisa, Pisa 1940; M. Pera, $L$ 'architettura minore del periodo romanico in Pisa. Le chiese diS. Frediano e di S. Michele degli Scalzi, Roma 1954.

${ }^{5}$ Con el término indicadores cronológicos se entienden los elementos que pueden aportar información sobre la cronología de la arquitectura; $c f r$. T. Mannoni, Metodi di datazione dell'edilizia storica, ArchMed, XI (1984), pp. 396-403.

${ }^{6}$ G. P. Brogiolo, Archeologia dell 'edilizia storica, Como 1988, p. 33; G. P. Brogiolo, Campionatura e obiettivi nell 'analisi stratigrafica degli elevati, in Archeologia e Restauro dei Monumenti, dirigida por R. Francovich-R. Parenti, Florencia 1988, pp. 339-340.

' R. Parenti, La aplicación del método estratigráfico para el análisis de monumentos, en las actas de las II Jornadas del patrimonio de Priego. Patrimonioy Ciudad. Priego de Córdoba, 26-29 de octubre de 1993, en prensa.

${ }^{8}$ G. P. Brogiolo, Archeologia dell 'edilizia, cit., p. 97.

${ }^{9} \mathrm{R}$. Parenti, Una proposta di classificazione tipologica delle murature post-classiche, en Conoscenze e sviluppi teorici per la conservazione disistemi costruttivitradizionali in muratura, Padua 1987, pp. 49-61, y R. Parenti, Sulle possibilità di datazione e di classificazione delle murature, en Archeologia e Restauro dei Monumenti, cit., pp. 280-304, con especial referencia a las obras de fábrica tradicionales.

${ }^{10}$ En este interesante campo se han obtenido resultados en Pisa, siendo el más importante la coordinación entre fuentes escritas $\mathrm{y}$ restos materiales de una edificación que ha dejado construcciones fundamentales que se fechan entre los siglos XI y XIV, cfr., F. Redi, Pisa, com'era: archeologia, urbanistica e strutture materiali (secoli V-XIV), Nápoles 1991.

${ }^{11}$ Lo hemos verificado en el análisis de todo un pueblo medieval abandonado -cfr. R. Parenti, Fonti materiali e lettura stratigrafica di un centro urbano: $i$ resultati de una sperimentazione "non tradizionale", ArchMed, XIX (1992), pp. 7-62- y lo estamos comprobando en Siena y en otros centros habitados.

${ }^{12}$ Ver nota ${ }^{9}$.

${ }^{13}$ S. Fossati, Possibilità di datare complessi dimattoni, ArchMed, XI (1984), p. 395; S. Fossati, La datazione dei mattoni: una proposta di metodo, ArchMed, XII (1985), pp. 731-736; T. Mannoni-M. Milanese, Mensiocronologia, en Archeologia e restauro dei Monumenti, cit., pp. 383-402; D. Pittaluga-P. Ghislanzoni, Mensiocronologia dei mattoni: la statistica applicata all'analisi, ArchMed, XVIII (1991), pp. 683-686.

${ }^{14}$ I. Ferrando Cabona-E. Crusi, Costruzioni rurali in Lunigiana; elementitipo ed evoluzione delle strutture insediative, ArchMed, VII (1980), pp. 247-270; I. Ferrando-T. Mannoni-R. Pagella, Cronotipologia, ArchMed, XVI (1989), pp. 647-661, y para algunos aspectos bastante innovadores, F. Gabrielli, Osservazioni di cronotipologia sulle aperture a sesto acuto della facciata dello Spedale, in Santa Maria della Scala. Archeologia ed edilizia sulla piazza dello Spadale, dirigido por E. Boldrini-R. Parenti, Florencia 1991, pp. 138-160.

${ }^{15}$ Esta técnica exigebuenos excavadores, algunos picapedreros, pero, sobre todo, buenos albañiles -T. Mannoni-A. CagnonaS. Falsini-P. Ghislanzoni-D. Pittaluga, Archeologia ed archeometria dei muri in pietra. Superfici e strutture in Liguria, p. 155, en La pietra nell 'architettura: Strutture e Superfici. Atti del convegno di Bressanone. 25-28 de junio de 1991, Padua 1991, pp. 151-162.

${ }^{16}$ Sicurezza e conservazione dei centri storici in area sismica. Il caso Ortigia, dirigido por A. Giuffrè, Bari-Roma 1993.

${ }^{17}$ Los resultados obtenidos en el análisis de la fachada del Spedale Santa Maria della Scala de Siena, la mayor superficie examinada hasta ahora en Europa con el instrumento estratigráfico, contestan convincentemente a algunas críticas promovidas por R. Bonelli. Cfr. Santa Maria della Scala, Archeologia ed edilizia, cit. y R. Bonelli, Archeologia stratigrafica e storia dell 'architettura, Architettura, Storia e documenti, 2 (1986), pp. 5-10.

${ }^{18}$ Para la descripción de los acontecimientos constructivos de un edificio en serie en San Giovanni Valdarno, véase R. Parenti, Massa Marittima e San Giovanni Valdarno: centri fondati e tipi edilizi. L'approccio archeologico, "Storia dell Città", 52 (1990), pp. 71-76; E. Boldrini-D. De Luca-R. Francovich, $L$ 'archeologia di una terra nuova. San Giovanni Valdarno, en Atti del Convegno I borghi nuovi. Secoli XII-XV. Cuneo, diciembre de 1990 , c.s.

${ }^{19}$ Además de ponencias presentadas en congresos muy recientes, una primera aportación ejemplar sobre los tipos de daños causados por los terremotos en los edificios religiosos se encuentra en Le chiese e il terremoto. Dalla vulnerabilità constatata nel terremoto del Friulial miglioramento antisismico nel restuaro, verso una politica di prevenzione, dirigido por $\mathrm{F}$. Doglioni-A. Moretti-V. Petrini, Trieste 1994. 